\title{
Isotropy Analysis of Parallel Six-Axis Accelerometer on Circular Hyperboloids
}

\author{
Chengxin Du, Jiaguo Tang, Chunzhan Yu*, Qin yin and Yili Zheng
}

Received: April 16, 2020. Revised: May 26, 2020. Accepted: June 5, 2020. Published: June 9, 2020

\begin{abstract}
This paper introduced the isotropic accelerometer using the circular hyperboloids method, which based on modified Gough-Stewart platform (GSP). By the static model of the accelerometer, the isotropy is defined on the acceleration matrix. On the basis of the isotropy condition, the relationship between isotropy index and geometric parameters of circular hyperboloids was investigated. Calculating the isotropy index by the optimization tool, this paper verified that it is feasible to achieve isotropy for the accelerometer. Then taking mass into account, a case is presented to optimize the parameters to construct isotropic accelerometer on circular hyperboloids. According to the 3D model of isotropic accelerometer, the static characteristic simulation was carried out by the finite element method. Based on the simulation experimental results, the calibration matrix was deduced, and the experimental isotropy index was obtained. Comparing the theoretical and experimental isotropy index, the method of circular hyperboloids was proved to be reliable and valid to construct isotropic accelerometer.
\end{abstract}

Keywords - Circular hyperboloids, isotropy, modified Gough-Ste wart platform, six-axis accelerometer, static model

\section{INTRODUCTION}

$\mathrm{A}$ CCELEROMETERS, which serve to measure accelerations in a space, have been widely used in numerous research areas such as inertial navigation [1], robot control [2], head impact sensors [3], estimating heart rate [4], fabricating complicated $3 \mathrm{D}$ devices [5], etc. How to fabricate the elastic structure is the key design of a multi-dimensional sensor. Due to the outstanding advantages in terms of precision, high stiffness, anti-interference, and compact structure, the Stewart platform [6] can be particularly applied to the elastic structure of six-axis accelerometers [7-10]. This paper introduced a small range isotropic accelerometer to accurately measure low frequency

This work was supported by the National Natural Science Foundation of the PR's of China (NSFC) (Grant number 31670719), and the National Students' Innovation Training Program (Grant number 201610022048). The authors are grateful to the project sponsored by the China Scholarship Council (CSC).

Chengxin Du, with Beijing Forestry University, Beijing, 100083 China,

Jiaguo Tang, with Beijing Forestry University, Beijing, 100083 China,

Chunzhan Yu, with Beijing Forestry University, Beijing, 100083 China,

Qin Yin, with Beijing Forestry University, Beijing, 100083 China,

Yili Zheng, with Beijing Forestry University, Beijing, 100083 China, and slowly change acceleration.

For multi-axis accelerometers, isotropy is one of the key factors to performance. Isotropy indicates that the accelerometer exhibits equal properties in all directions [11], and it usually leads to the relative minimum error [12]. The isotropy performance of the accelerometer with a parallel mechanism mainly depends on the isotropy performance of the parallel mechanism. Designing a parallel robot with isotropy in one pose and position or over its full workspace is often considered as a design objective [13-15]. Zanganeh and Angele [16] defined a set of conditions, and presented a systematic method to design a 6-DOF isotropic parallel manipulator. Legnani introduced the concept of isotropy of a generic parallel manipulator, and presented how to design an isotropic parallel manipulator by changing the location of the Tool Center Point [17]. Tsai presented a reliable method to develop an isotropic parallel manipulator by using an isotropy generator, which consists of six straight lines achieving the isotropy conditions [15]. The optimal isotropy design for a multi-dimensional sensor is one of the most significant issues in this field of research. In the literature of multi-dimensional sensor design, the elastic structures of the sensor have been generally based on a standard Gough-Stewart platform (GSP). According to the physical model of solution space, Wang [18] and $\mathrm{Yu}$ [19] conducted an optimal design by using the isotropy performance atlases. Afzali-Far proposed full-parametric solutions to obtain isotropy based on two kinematic arrangements [20]. Hou applied genetic algorithms to optimize the parameters of the hyperstatic six-component force/torque sensor [21] for improving the isotropy. Jiang proposed the method of circular hyperboloid for the standard GSP, which presented a new approach for isotropy [22]. Tong applied the method of circular hyperboloid for satisfying the isotropy [23]. However, a drawback for standard GSP is that it is impossible to achieve isotropy [14, 23-25].

Modified GSP is feasible to satisfy isotropy [26]. Simultaneously, a broader definition includes more configurations to be selected than standard GSP. Jafari proposed a method to design a micromanipulator based on orthogonal GSP (a sort of modified GSP), which can meet isotropy over a small workspace [27]. Based on the screw theory, Yao derived the relations of key structural parameters leading to an isotropic configuration of the force sensor [11]. 
Hou proposed a force sensor where both the force isotropy index and torque isotropy index surpassed 0.9 , and built the solid model of the elastic structure [21,28]. Additionally, the method of circular hyperboloids can be used to optimize the parameters of sensors based on modified GSP. Jiang constructed the mathematical model of modified GSP according to the method of circular hyperboloids, and deduced the relations between isotropy and the geometric parameters of hyperboloids [29]. This was a novel approach to achieve isotropy with simplified design steps. Tong $[23,26]$ applied the method of circular hyperboloids to study the multi-dimensional sensor and proved that it could achieve isotropy for multi-dimensional sensors based on modified GSP.

The main contributions focus on three folds:

(1) Based on the parallel mechanism theory and circular hyperboloids method, the relationship between isotropy index and geometric parameters of circular hyperboloids was investigated.

(2) Applied circular hyperboloids method, the influence factors of isotropy for the accelerometer based on equal-leg modified GSP were analyzed, and complete isotropy for the accelerometer on determined circular hyperboloids proved to be achieved.

(3) Considering the factors of mass such as: shape, dimension and material etc., the parameters of accelerometer were optimized on circular hyperboloids method to construct isotropic accelerometer, and feasibility of circular hyperboloids method were verified by simulation experiment.

The organization of this paper is as follows. Following the introduction, Section II presents the modified GSP and introduces the equal-leg modified GSP. In Section III, the static model is established, and the isotropy is defined. Section IV derives and investigates the relationship between isotropy index and geometric parameters of hyperboloids. In Section V, the way to verify the isotropy based on the method of circular hyperboloids is presented, and the isotropy for accelerometer based on equal-leg modified GSP is proven. Section VIprovide an example applies to illustrate the design process of an isotropic accelerometer. In Section VII the relationship between acceleration and deformation along each leg is derived, and the calibration matrix is introduced. According to the solid model on the example's structure, the static characteristic simulation was carried out. Meanwhile, the isotropy index of the simulation experiments was yielded. Finally, the conclusion is drawn in Section VIII

\section{MODIFIED GSP}

\section{A. Modified GSP Based on Circular Hyperboloids}

A modified GSP consists of a lower platform, an upper platform, and six supporting legs. The upper platform and lower platform are composed of two concentric circles, the outer one and the inner one. As shown in Figure 1, six legs are divided into two groups, the outer legs and the inner legs, and each group of legs rotates symmetrically along the z-axis, with the step of
$2 \pi / 3$. The outer legs are linked to the outer concentric circles by the outer hinge $A_{i}$ and $B_{i}(i=1,3,5)$. Similarly, the inner legs are linked to the inner concentric circle by the inner hinge $A_{i}$ and $B_{i}(i=2,4,6) . \alpha$ denotes the angle between the line of hinge $A_{1}$ or $A_{6}$ to the centre of the upper platform and the $\mathrm{x}$-axis.

In order to measure the acceleration on the parallel six-axis accelerometer, the structure is constructed as follow. The lower platform is used to detachably connect the carrier. The upper platform is the mass, which can transfer the generalized acceleration into inertial force. The hinges link the legs to the upper/lower platform and transmit force. Thus, the stress and strain can be caused in the legs made of elastic material and be measured by the strain gauge pasted on the legs.

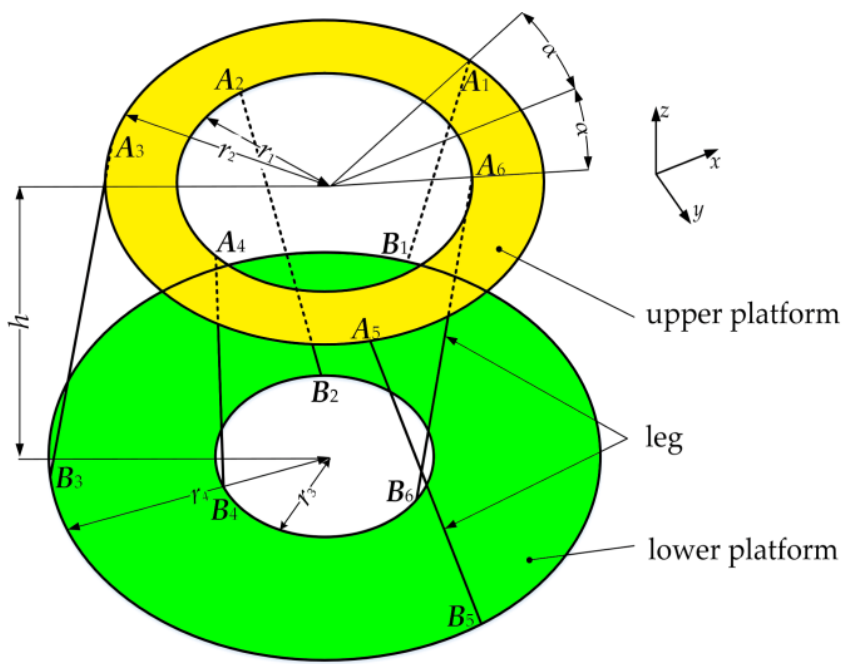

Fig. 1 Schematic diagram of the modified Gough-Stewart platform.

In the Cartesian coordinate system, the surface equations of circular hyperboloids are described as $[23,26,29]$ :

$$
\begin{aligned}
& D_{1}: \frac{x^{2}+y^{2}}{a_{1}^{2}}-\frac{\left(z-z_{1}\right)^{2}}{c_{1}^{2}}=1 \\
& D_{2}: \frac{x^{2}+y^{2}}{a_{2}^{2}}-\frac{\left(z-z_{2}\right)^{2}}{c_{2}^{2}}=1
\end{aligned}
$$

where: $a_{i}(i=1,2)$, denotes the waist radius of circular hyperboloids; $z_{i}(i=1,2)$ denotes the distance from the center of the circular hyperboloid waist circle to the origin of the coordinate; and $c_{i}(i=1,2)$ denotes the characteristic parameters of circular hyperboloids.

According to the structural parameters of circular hyperboloids [29,30], the unit vectors of the two generators of circular hyperboloids are:

$$
\begin{aligned}
& \boldsymbol{l}_{1}=\left(\begin{array}{lll}
x_{1} & y_{1} & z_{1}
\end{array}\right)=\left(\begin{array}{lll}
-t_{1} \sin \alpha & t_{1} \cos \alpha & u_{1}
\end{array}\right) \\
& \boldsymbol{l}_{2}=\left(\begin{array}{llll}
x_{2} & y_{2} & z_{2}
\end{array}\right)=\left(\begin{array}{lll}
-t_{2} \sin \alpha & t_{2} \cos \alpha & u_{2}
\end{array}\right)
\end{aligned}
$$

where $t_{i}(i=1,2)$ and $u_{i}(i=1,2)$ denote the characteristic parameters of the generators, respectively.

Here: 


$$
\begin{aligned}
& t_{i}=\frac{a_{i}}{\sqrt{a_{i}^{2}+c_{i}^{2}}} \\
& u_{i}=\frac{c_{i}}{\sqrt{a_{i}^{2}+c_{i}^{2}}}
\end{aligned}
$$

From the properties of circular hyperboloids [30], we can calculate the Force matrix of the accelerometer based on modified GSP [26, 29].

$$
\boldsymbol{J}^{-1}=\left(\begin{array}{c}
p_{1}(\alpha) \\
p_{2}(\alpha) \\
p_{1}\left(\alpha+\frac{2}{3} \pi\right) \\
p_{2}\left(\alpha+\frac{2}{3} \pi\right) \\
p_{1}\left(\alpha-\frac{2}{3} \pi\right) \\
p_{2}\left(\alpha-\frac{2}{3} \pi\right)
\end{array}\right)
$$

where $\boldsymbol{J}$ is the Force matrix of the accelerometer based on modified GSP, which represents the force transfer from the upper platform to each leg.

The vectors of $\boldsymbol{p}_{1}(\theta)$ and $\boldsymbol{p}_{2}(\theta)$ denote the unitary Plücker coordinates of the axes of No.1 and No.6 legs, respectively, which are functions of the rotation angle $\theta$ about the z-axis [29].

$$
\begin{aligned}
& \boldsymbol{p}_{1}(\theta)=\left(\begin{array}{c}
-t_{1} \sin \theta \\
t_{1} \cos \theta \\
u_{1} \\
-z_{1} t_{1} \cos \theta+a_{1} u_{1} \sin \theta \\
-z_{1} t_{1} \sin \theta-a_{1} u_{1} \cos \theta \\
a_{1} t_{1}
\end{array}\right) \\
& \boldsymbol{p}_{2}(\theta)=\left(\begin{array}{c}
-t_{2} \sin \theta \\
t_{2} \cos \theta \\
u_{2} \\
-z_{2} t_{2} \cos \theta+a_{2} u_{2} \sin \theta \\
-z_{2} t_{2} \sin \theta-a_{2} u_{2} \cos \theta \\
a_{2} t_{2}
\end{array}\right)
\end{aligned}
$$

\section{B. Equal-Leg Modified GSP}

Equal-leg modified GSP is a sort of modified GSP that has a simpler structure. Yao [11] proved that under the condition of isotropy, the upper hinge points $A_{i}(i=1,2,3,4,5,6)$ were distributed on a circle (shown in Figure.2). In other words, when the accelerometer based on equal-leg modified GSP satisfies isotropy, the two concentric circles in the upper platform have the same radius. The other definition of equal-leg modified GSP is the same as in Section 2.1.

\section{STATIC MODEL}

\section{A. Acceleration Matrix}

The static mathematical model of the parallel accelerometer is expressed as $[23,26]$ :

$$
\boldsymbol{J}^{T} f=\boldsymbol{M} \ddot{x}
$$

where $\ddot{x}$ is the generalized acceleration loaded on the mass.

Rewrite Equation (10):

$$
\ddot{x}=\boldsymbol{M}^{-1} \boldsymbol{J}^{T} f
$$

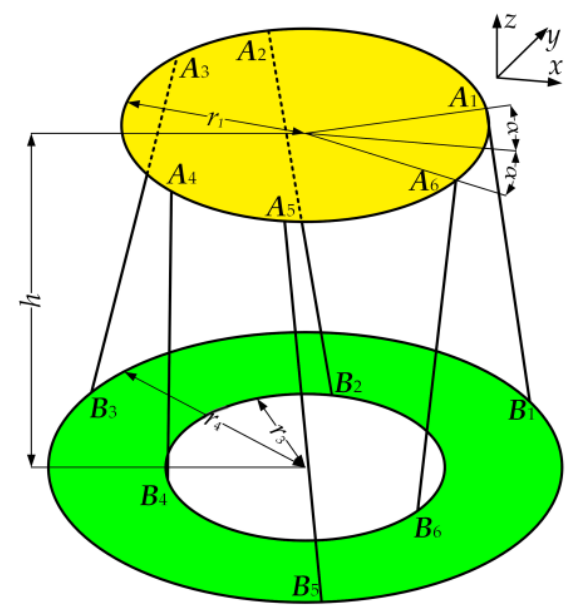

Fig. 2 Schematic diagram of the equal-leg modified Gough-Stewart platform.

where $\boldsymbol{f}=\left(f_{1} f_{2} f_{3} f_{4} f_{5} f_{6}\right)^{T}$ is the vector composed of the forces of the six legs, and $\boldsymbol{M}$ is the generalized mass.

Here:

$$
\boldsymbol{M}=\operatorname{diag}\left[\begin{array}{lllllll}
m & m & m & I_{x} & I_{y} & I_{z}
\end{array}\right]
$$

where $I_{x}, I_{y}$ and $I_{z}$ are the moments of inertia along each axis, and $m$ is the mass.

According to the definition in [26], the acceleration matrix of the accelerometer can be written as:

$$
\boldsymbol{G}=\boldsymbol{M}^{-1} \boldsymbol{J}^{-T}
$$

\section{B. Isotropy}

The isotropy of a parallel accelerometer depends on the isotropy of the structure. Isotropy indicates that the six-axis accelerometer should have equal properties in all measuring dimensions in order to obtain the minimum measurement error. During designing and manufacturing for the structure of parallel accelerometer, there are structural errors $\Delta \boldsymbol{G}$ causing errors $\Delta \ddot{x}$ in measuring acceleration The relationship between $\Delta \boldsymbol{G}$ and $\Delta \ddot{x}$ is shown in Equation (14) [31,32]:

$$
\frac{\|\Delta \ddot{x}\|}{\|\ddot{x}\|} \leq\left\|\boldsymbol{G}^{-1}\right\|\|\boldsymbol{G}\| \frac{\|\Delta \boldsymbol{G}\|}{\|\boldsymbol{G}\|}
$$

Then, the error amplification factor, which is called the condition number $\mu[31,33]$, is expressed as:

$$
\mu=\operatorname{cond}(\boldsymbol{G})=\left\|\boldsymbol{G}^{-1}|\|\mid \boldsymbol{G}\|\right.
$$

The condition number is bounded to the range $1 \leq \mu<\infty$. 
The reciprocal of the condition number, which is called the isotropy index $[19,32,34,35]$, is often used. It can be written as:

$$
S=\frac{1}{\operatorname{cond}(\boldsymbol{G})}
$$

The range of isotropy index is within $[0,1]$. When the condition number equals 1 , the accelerometer is considered as most slightly affected by the errors in the elastic structure, and the accelerometer is isotropy.

As is widely known, the linear acceleration and the angular acceleration have different dimension. So it is meaningless in physics to calculate isotropy between the linear acceleration and the angular acceleration [11]. In the paper, we discuss linear isotropy and angular isotropy, respectively [36]. Rewriting the acceleration matrix:

$$
\boldsymbol{G}=\left(\begin{array}{ll}
\boldsymbol{G}_{a} & \boldsymbol{G}_{\varepsilon}
\end{array}\right)^{T}
$$

where $\boldsymbol{G}_{a}$ is the linear acceleration matrix, and $\boldsymbol{G}_{\varsigma}$ is the angular acceleration matrix.

Then, the linear isotropy is evaluated by the linear isotropy index:

$$
S_{a}=\frac{1}{\operatorname{cond}\left(\boldsymbol{G}_{a}\right)}
$$

The angular isotropy is evaluated by the angular isotropy index:

$$
S_{\varsigma}=\frac{1}{\operatorname{cond}\left(\boldsymbol{G}_{\varsigma}\right)}
$$

Under the same definition, the linear/angular isotropy index is within the range $[0,1]$, and the condition $S_{a}=1$ or $S_{\varsigma}=1$ is called linear or angular isotropy. When the condition is $S_{a}=1$ and $S_{\varsigma}=1$, it is called isotropy for the parallel six-axis accelerometer [32].

\section{RELATIONSHIP BETWEEN ISOTROPY AND GEOMETRIC PARAMETERS OF CIRCULAR HYPERBOLOIDS}

In the method of circular hyperboloids, the structure of the parallel six-axis accelerometer is constructed from circular hyperboloids [22,29]. In order to obtain the isotropic accelerometer, the geometric parameters of circular hyperboloids are constrained under isotropy conditions.

For convenience, define the ratio of the waist radius $n$.

$$
n=\frac{a_{1}}{a_{2}}
$$

where $n$ changes within the range $0 \leq n \leq 1$, the value of $n$ determines the family of circular hyperboloids. When $n=1$, the two circular hyperboloids are parallel to each other, and can make the structure of accelerometer more compact. Moreover, only if these two circular hyperboloids are parallel to each other, the equal-leg modified GSP can be fabricated.

In order to achieve isotropy, the parameters of the circular hyperboloids need to satisfy the prerequisites of isotropy [29].

$$
\begin{aligned}
a_{1} t_{1} u_{1} & =a_{2} t_{2} u_{2} \\
t_{1}^{2} z_{1} & =t_{2}^{2} z_{2}
\end{aligned}
$$

When the accelerometer achieves linear isotropy, the parameters must satisfy the conditions as follows:

$$
\begin{aligned}
& u_{1}^{2}+u_{2}^{2}=\frac{2}{3} \\
& t_{1}^{2}+t_{2}^{2}=\frac{4}{3}
\end{aligned}
$$

In addition, the length of unit vector for generatrix $\left\|l_{i}\right\|=1(\mathrm{~mm})$, which can be expressed as:

$$
t_{i}^{2}+u_{i}^{2}=1
$$

From Equations (20), (21), (23), and (25), it leads to

$$
\left(n^{2}-1\right) u^{4}+\left(\frac{1}{3}-n^{2}\right) u_{1}^{2}+\frac{2}{9}=0
$$

When the accelerometer achieves angular isotropy, the parameters must satisfy the conditions as follows [29]:

$$
\begin{gathered}
a_{1}^{2} t_{1}^{2}+a_{2}^{2} t_{2}^{2}=\frac{2 I_{z}}{3 m} \\
a_{1}^{2} u_{1}^{2}+z_{1}^{2} t_{1}^{2}+a_{2}^{2} u_{2}^{2}+z_{2}^{2} t_{2}^{2}=\frac{4 I_{x}}{3 m}
\end{gathered}
$$

Substituting Equation (21), Equation (23), and Equation (25) into Equation (27) produces:

$$
a_{2}^{2}=\frac{I_{z} u_{1}^{2}}{m\left(u_{1}^{2}+\frac{1}{3}\right)}
$$

Inserting Equation (23) and Equation (25) into Equation (22), we have:

$$
z_{1}=\frac{\left(u_{1}^{2}+\frac{1}{3}\right) z_{2}}{1-u_{1}^{2}}
$$

Substituting Equation (21), Equation (23), Equation (25) and Equations (29) and (30) into Equation (28), we can obtain:

$$
z_{2}^{2}=\frac{3\left(1-u_{1}^{2}\right)}{4\left(u_{1}^{2}+\frac{1}{3}\right)}\left(\frac{4 I_{x}}{3 m}-\frac{I_{z} u_{1}^{2}\left(\frac{2}{3}+\left(n^{2}-1\right) u_{1}^{2}\right)}{m\left(u_{1}^{2}+\frac{1}{3}\right)}\right)
$$

Combining Equation (5) and Equation (6) produces:

$$
\begin{aligned}
& c_{1}^{2}=\frac{u_{1}^{2} a_{1}^{2}}{t_{1}^{2}} \\
& c_{2}^{2}=\frac{u_{2}^{2} a_{2}^{2}}{t_{2}^{2}}
\end{aligned}
$$

Set the parameters $\alpha$ and $n$, give the moments of inertia $I_{x}, I_{y}, I_{z}$ and mass $m$, then the surface equations of the circular hyperboloids can be determined.

\section{ISOTROPY VERIFICATION}

\section{A. Steps of Verification}

In order to verify the validity of the isotropy condition, it is necessary to calculate the isotropy index. Moreover, different accelerometers located on the same circular hyperboloids have the same acceleration matrix. So long as the parameters of circular hyperboloids satisfy isotropy condition, the accelerometers constructed from the circular hyperboloids are 
isotropy.

The verification steps are given as follows:

(1). Given the generalized mass and the determined parameters $n$ and $\alpha$, the geometric parameters for target circular hyperboloids can be calculated under isotropy condition.

(2). Calculate the acceleration matrix with the geometric parameters of target circular hyperboloids and Equation (13). Then, the isotropy index is computed by Equations (17) - (19).

\section{B. Verification for Equal-Leg Modified GSPs}

Equal-leg modified GSP is more suitable to construct the accelerometer for its uniform output. In order to calculate the isotropy index of the accelerometer based on equal-leg modified GSP, let $n=1$. Additionally, we used the mass shaped cylinder with radius $R$, height $h=\sqrt{3} R$ and density of the cylindrical mass $\rho=8900 \mathrm{~kg} / \mathrm{m}^{3}$. Set the radius of the mass $R$ as a variable, the isotropy indexes $S_{a}$ and $S_{\varsigma}$ are related to the radius.

In order to be easy to calculate isotropy indexes $S_{a}$ and $S_{\varsigma}$, let the radius $R$ in the range of $0.01 m \leq R \leq 0.08 m$ and the step length $\Delta R=0.0001 \mathrm{~m}$. $\alpha$ was chosen arbitrarily within the range of $0 \leq \alpha \leq \pi / 6$. We used the optimization tool to obtain the relations, which are shown in Figure 3.

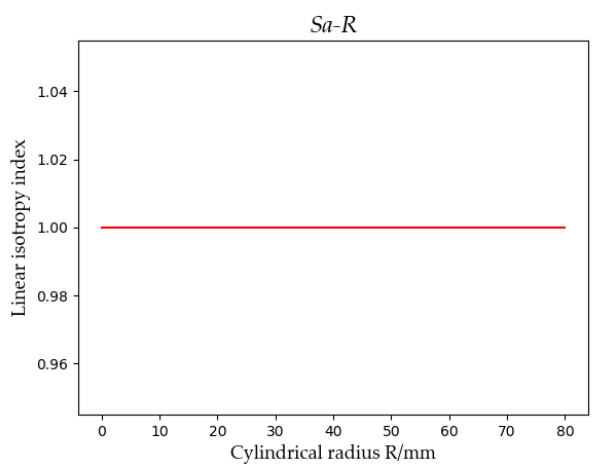

(a)

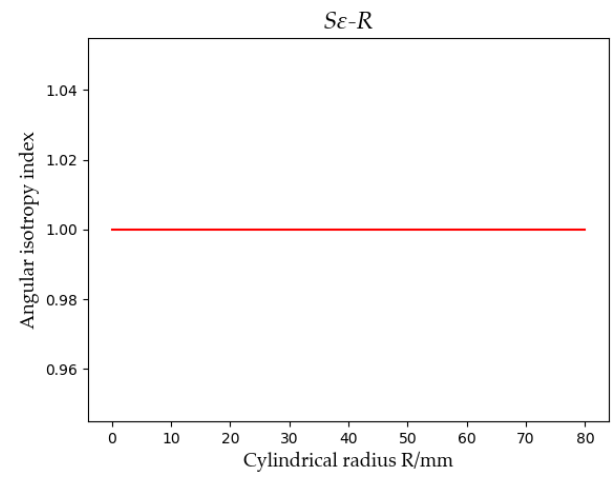

(b)

Fig. 3 Relationship between $S_{a}, S_{b}$ and $R$. (a) $S_{a}-R$; and (b) $S_{\varsigma}-R$.

Similarly, let the angle $\alpha$ in the range of $0 \leq \alpha \leq \pi / 6$ and the step length $\Delta \alpha=\pi / 3000$, radius $R$ is chosen arbitrarily. We used the optimization tool to obtain the relationship between the isotropy indexes and the angle. Furthermore, the relations are shown in Figure 4.

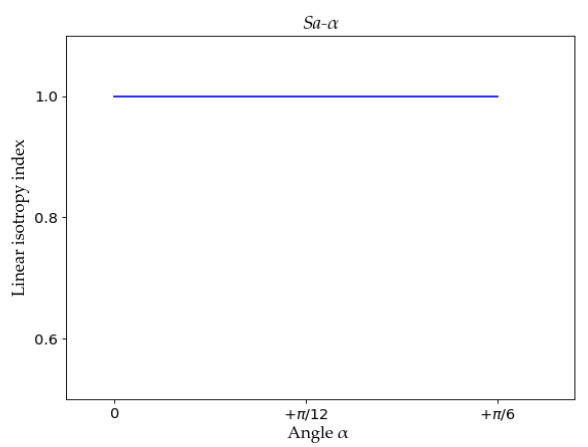

(a)

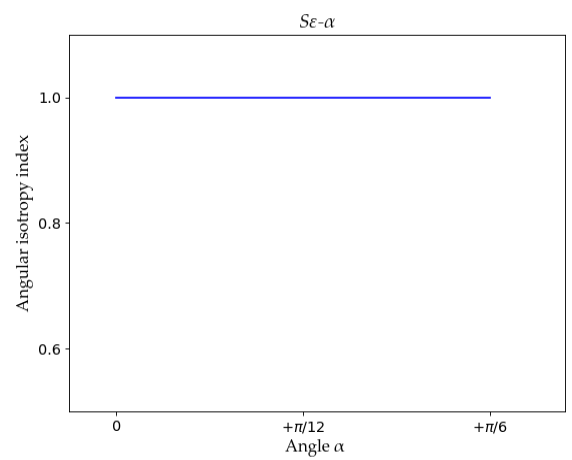

(b)

Fig. 4 Relationship between $S_{a}, S_{b}$ and $\alpha$. (a) $S_{a}-\alpha$; and (b) $S_{\varsigma}-\alpha$.

Based on Figures 3 and Figures 4, we can draw conclusions as follows:

1) With the change of the variable $R$ or $\alpha$, the linear and angular isotropy index remain constant 1 , which is isotropy for the parallel six-axis accelerometer.

2) As a matter of fact, Figure 3 represents a series of circular hyperboloids with isotropic condition and Figure 4 represents a serious of isotropic accelerometer on determined circular hyperboloids.

\section{EXAMPLE FOR ISOTROPIC ACCELEROMETER}

\section{A. Parameters of circular hyperboloids}

An example is applied to illustrate the design process of an isotropic accelerometer. The steps are presented in detail as follows:

1) Choose the ratio of the waist radius $n$

The ratio of the waist radius within the range $0 \leq n \leq 1$. In order to establish the accelerometer based on equal-leg modified GSP, let $n=1$.

2) Give the angle $\alpha$

The angel $\alpha$ is in the range $0 \leq \alpha \leq \pi / 6$. Considering the interference between mass and other parts and installation of the accelerometer, $\alpha$ takes the maximum $\pi / 6$.

3) Set the generalized mass 
The parameters of the cylindrical mass include the radius $R=30 \mathrm{~mm}$, height $h=\sqrt{3} R$, density of the mass $\rho=8900 \mathrm{~kg} / \mathrm{m}^{3}$, mass $m=\rho \pi R^{2} h$, and moment of inertia $I_{x}=I_{y}=I_{z}=m R^{2} / 2$.

From Equations (19)-(32) and the relevant data, we can calculate the geometry parameters of the target circular hyperboloids:

$$
\begin{gathered}
a_{1}=a_{2}=15 \mathrm{~mm}, z_{1}=z_{2}=18.4 \mathrm{~mm}, \\
c_{1}^{2}=c_{2}^{2}=112.5 \mathrm{~mm}^{2}
\end{gathered}
$$

Then, the surface equations of the target circular hyperboloids (as shown in Figure 5) can be determined:

$$
D_{1}: \frac{x^{2}+y^{2}}{225}-\frac{(z-18.4)^{2}}{112.5}=1 D_{2}: \frac{x^{2}+y^{2}}{225}-\frac{(z+18.4)^{2}}{112.5}=1
$$

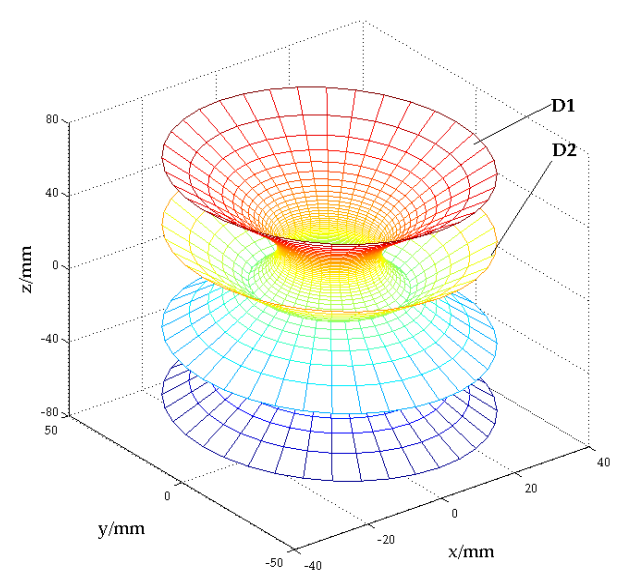

Fig. 5 Target circular hyperboloids.

\section{B. Structure Established from Circular Hyperboloids}

The example ( $R=0.03 \mathrm{~mm}, \alpha=\pi / 6)$ is included in the isotropy verification range in section 5 . Thus, the structures of the accelerometer generated from the target circular hyperboloids are isotropic. The goal of this section was to establish the structure of the accelerometer.

In the Cartesian coordinate system, first, two planes are selected that are parallel with the XOY plane to intersect the circular hyperboloids, then the planes for the upper platform and lower platform are determined respectively [23]. In order to establish the accelerometer based on equal-leg modified GSP, the plane for the upper platform should coincide with the XOY plane. The plane for lower platform is determined by the height $h_{r}$, which is the height between the upper platform and lower platform. Two planes and circular hyperboloids intersect at four circulars, where the radiuses are $r_{1}, r_{2}, r_{3}$, and $r_{4}$.

As the structure of accelerometer lies on circular hyperboloids, each group of legs coincides with the generatrices of the circular hyperboloid, respectively. $\alpha$ in Figure 6 can define the position of two initial legs in each circular hyperboloid, and its range is $[0, \pi / 6]$. In order to avoid the interference in each leg, $\alpha$ takes the maximum. By rotating the two initial legs with the step $2 \pi / 3$ along the $z$-axis, other two groups of legs are obtained. And the structure of the accelerometer is fabricated and is shown in Figure 6. The structural parameters' values are shown in Table 1.

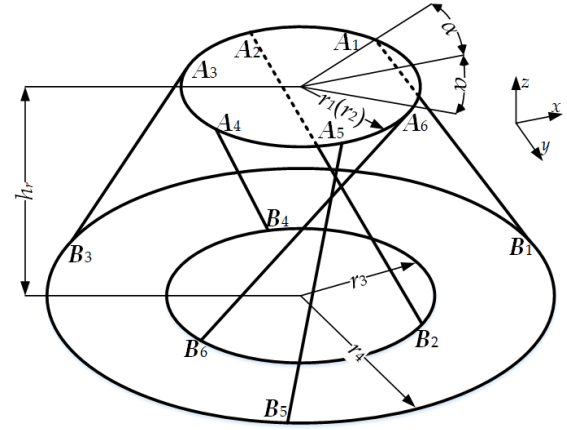

Fig. 6 Structure of the example.

Table 1. Structural parameters' values of the example.

\begin{tabular}{cc}
\hline Parameters & Value \\
\hline$r_{1}$ & $30.000 \mathrm{~mm}$ \\
$r_{2}$ & $30.000 \mathrm{~mm}$ \\
$r_{3}$ & $31.500 \mathrm{~mm}$ \\
$r_{4}$ & $81.150 \mathrm{~mm}$ \\
$\alpha$ & $\pi / 6 \quad \mathrm{rad}$ \\
$h_{r}$ & $38.000 \mathrm{~mm}$ \\
\hline
\end{tabular}

\section{SIMULATION EXPERIMENT}

\section{A. Stiffness and Deformation}

According to the mechanics of materials, the relation between the forces and deformations on each leg can be expressed as:

$$
\boldsymbol{f}=\boldsymbol{K} \cdot \Delta \boldsymbol{l}
$$

where $\boldsymbol{K}$ is the diagonal stiffness matrix of the legs, which can be written as:

$$
\boldsymbol{K}=\operatorname{diag}\left(k_{1}, k_{2}, k_{3}, k_{4}, k_{5}, k_{6}\right)
$$

Assuming the material and cross sectional area of each leg are the same, the stiffness coefficient $k_{i}$ satisfies:

$$
k_{i}=E A / l_{i}
$$

where $E$ is the modulus of elasticity of the legs; $A$ is the cross sectional area of the legs; and $l_{i}(i=1,2,3,4,5,6)$ is the length of each leg.

The relation between the forces and the strain on the legs can be derived from Equations (34) - (36) as following:

$$
\boldsymbol{f}=E A \boldsymbol{q}
$$

where $q=\left(q_{1}, q_{2}, q_{3}, q_{4}, q_{5}, q_{6}\right)^{T}$ is the vector composed of the strain of six legs.

Substituting Equation (13) and Equation (37) into Equation (11), the relation between acceleration and strain on each leg is conducted:

$$
\ddot{x}=E A \boldsymbol{G}
$$

\section{B. Calibration Matrix}

In the practical measurement, the elastic structure of a six-axis accelerometer outputs the strain on the legs instead of the acceleration. Assuming a single direction acceleration 
vector is loading on the accelerometer, the strain output from each leg contains the coupling strain between the axes. In order to decouple the coupling strain, we introduce the calibration matrix. The relationship between the input and output of the accelerometer can be expressed [32]

$$
\boldsymbol{\omega}=\boldsymbol{\beta} \boldsymbol{V}
$$

where

$$
\left.\omega=\mid \begin{array}{cccc}
\omega_{x 1} & \omega_{x 2} & \ldots & \omega_{x i} \\
\omega_{y 1} & \omega_{y 2} & \ldots & \omega_{y i} \\
\omega_{z 1} & \omega_{z 2} & \ldots & \omega_{z i} \\
\sigma_{x 1} & \sigma_{x 2} & \ldots & \sigma_{x i} \\
\sigma_{y 1} & \sigma_{y 2} & \ldots & \sigma_{y i} \\
\sigma_{z 1} & \sigma_{z 2} & \ldots & \sigma_{z i}
\end{array}\right\rfloor
$$

is the acceleration vector for multi-times loading in a single direction. $\omega_{x i}, \omega_{y i}, \omega_{z i}$ are the three linear acceleration components and $\sigma_{x i}, \sigma_{y i}, \sigma_{z i}$ are the three angular acceleration components.

$$
\beta=\left[\begin{array}{cccc}
\beta_{11} & \beta_{12} & \ldots & \beta_{16} \\
\beta_{21} & \beta_{22} & \ldots & \beta_{26} \\
\vdots & \vdots & \ddots & \vdots \\
\beta_{61} & \beta_{62} & \ldots & \beta_{66}
\end{array}\right\rfloor
$$

is the calibration matrix of the accelerometer, the first three rows of the calibration matrix is the linear acceleration calibration matrix, and the last three rows of the calibration matrix is the angular acceleration calibration matrix.

$\boldsymbol{V}$ is the strain on each leg corresponding to the multi-times loading acceleration vector. Rewriting Equation (39), the calibration matrix of the accelerometer can be expressed as:

$$
\boldsymbol{\beta}=\omega \boldsymbol{V}^{-1}
$$

where if $\boldsymbol{V}$ is not a square matrix, the inverse of $\boldsymbol{V}$ is called its generalized inverse matrix.

Calibration matrix $\boldsymbol{\beta}$ reflects the relationship between the acceleration vector and strain, which are affected by the measuring accuracy and coupling error.

\section{Simulation Experiment}

The elastic structure of the accelerometer is the key to transform the acceleration into strain. Compared with the traditional spherical hinge, flexure hinge have the following main advantages: decreased assembly requirements, no friction, reduce weight, and no lubrication [37-39]. Therefore, we applied the flexure hinge to construct the solid model on the example's structure for the accelerometer, where the leg's cross sectional area $A=1.6 e-5 m^{2}$, the diameter of flexure hinge $d_{h}=3 \mathrm{~mm}$, and the length of the flexure hinge $l_{h}=4 \mathrm{~mm}$.

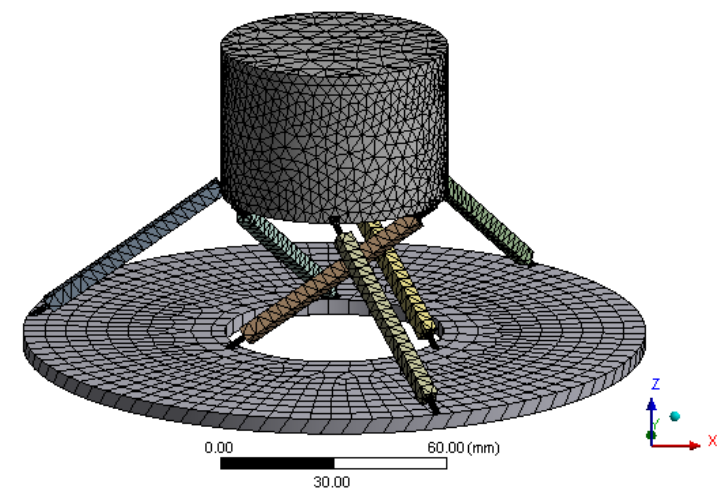

Fig. 7 The finite element model of the accelerometer.

The finite element model is shown in Figure 7. The material of the mass was a copper alloy, with the density of $8900 \mathrm{~kg} / \mathrm{m}^{3}$.

The material of the leg was an aluminum alloy, the density was $2700 \mathrm{~kg} / \mathrm{m}^{3}$, the elastic modulus was 7.1E10Pa, and the

Poisson's ratio was 0.31 . Additionally, the static characteristic simulation of the accelerometer was carried out by the finite element method.

Setting the fixed constraint on the lower platform and loading the acceleration along each axis for multi-times respectively, then the acceleration for multi-times loading can be written as:

$$
\boldsymbol{\omega}_{0}=\left[\begin{array}{cccccc}
\omega_{x 1} & 0 & 0 & 0 & 0 & 0 \\
0 & \omega_{y 2} & 0 & 0 & 0 & 0 \\
0 & 0 & \omega_{z 3} & 0 & 0 & 0 \\
0 & 0 & 0 & \sigma_{x 4} & 0 & 0 \\
0 & 0 & 0 & 0 & \sigma_{y 5} & 0 \\
0 & 0 & 0 & 0 & 0 & \sigma_{z 6}
\end{array}\right]
$$

Setting the linear acceleration along each axis to $9.8 \mathrm{~m} / \mathrm{s}^{2}$, the angular acceleration along each axis to $500 \mathrm{rad} / \mathrm{s}^{2}$, then $w_{x 1}=w_{y 2}=w_{z 3}=9.8 \mathrm{~m} / \mathrm{s}^{2}, \quad \sigma_{x 4}=\sigma_{y 5}=\sigma_{z 6}=500 \mathrm{rad} / \mathrm{s}^{2}$. The experimental strain on each leg can be obtained by a running simulation. The deformations of the six legs are shown in Figure 8.

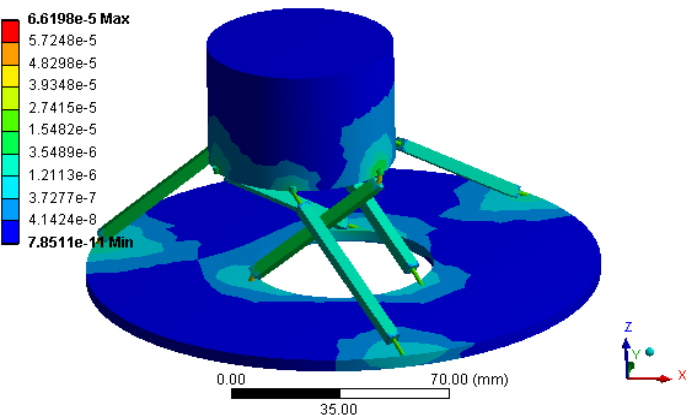

(a) 


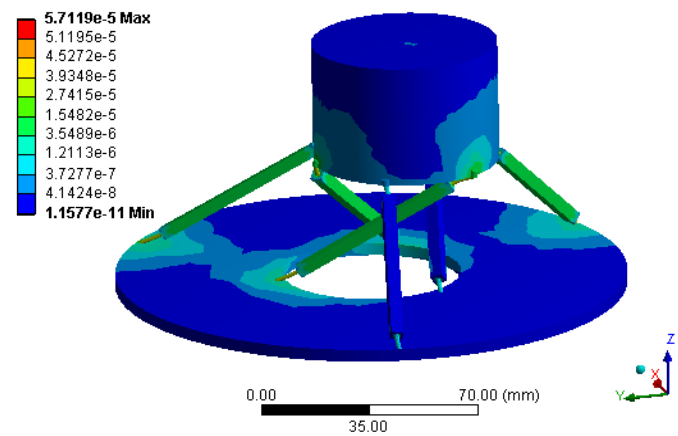

(b)

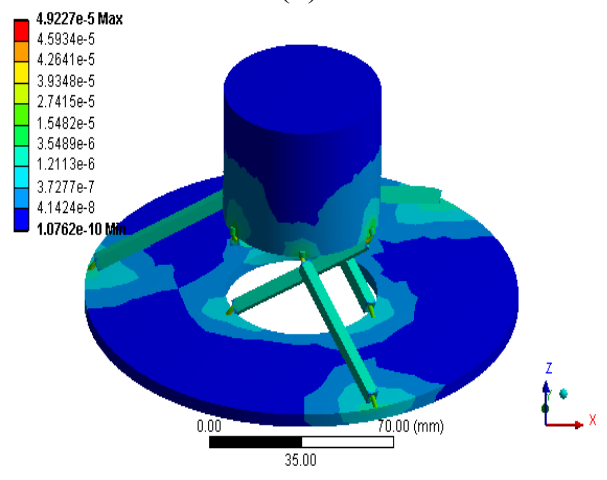

(c)

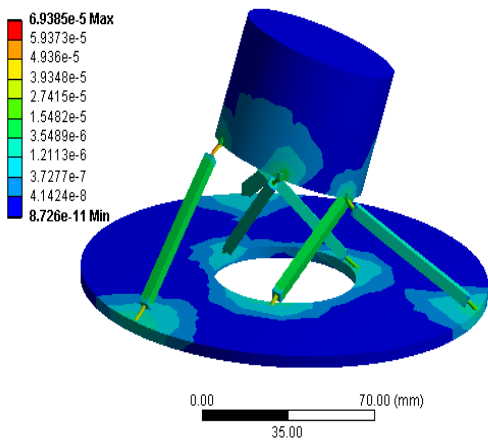

(d)

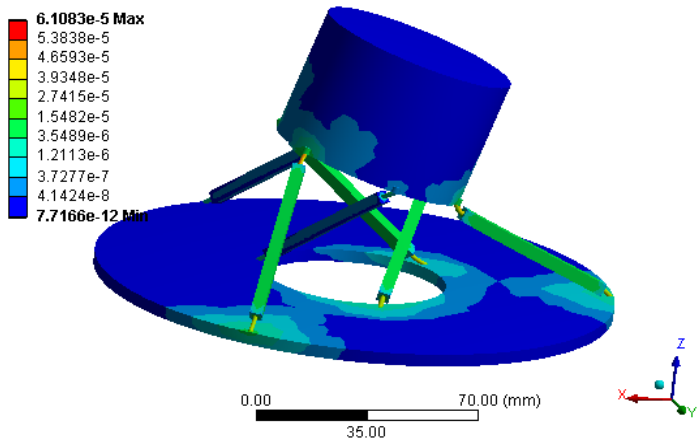

(e)

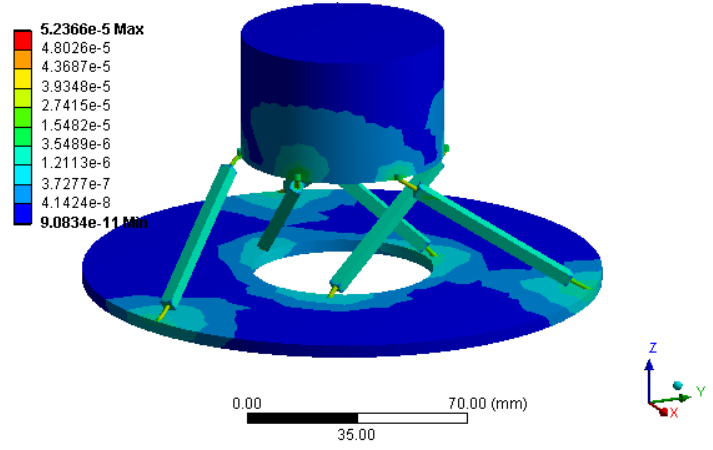

(f)

Fig. 8 Deformation of six legs on multi-times loading along each axis respectively. (a) deformation of six legs under a linear acceleration vector along the x-axis; (b) deformation of six legs under a linear acceleration vector along the y-axis; (c) deformation of six legs under a linear acceleration vector along the z-axis; (d) deformation of six legs under an angular acceleration vector along the $\mathrm{x}$-axis; (e) deformation of six legs under an angular acceleration vector along the y-axis; and (f) deformation of six legs under an angular acceleration vector along the z-axis.

The strain matrix is obtained from the experiment:

$$
\boldsymbol{V}=\left[\begin{array}{cccccc}
2.141 & 3.704 & -3.208 & 2.368 & -4.706 & -3.329 \\
-4.304 & 0.008 & -3.020 & -2.262 & -4.087 & 3.357 \\
2.153 & -3.737 & -3.017 & -4.608 & 0.089 & -3.362 \\
2.151 & 3.707 & -3.019 & -2.443 & 4.081 & 3.359 \\
-4.294 & 0.009 & -3.022 & 2.374 & 3.993 & -3.365 \\
2.142 & -3.706 & -3.022 & 4.708 & 0.091 & 3.358
\end{array}\right] \times 10^{-6}
$$

\section{Comparison}

In order to validate the structure and the results of experiment, we compared the experimental isotropy index with the theoretical isotropy index.

The acceleration matrix of the accelerometer was obtained by the relevant parameters and Equation (13)

$\boldsymbol{G}_{1}=\left[\begin{array}{cccccc}-0.335 & 0.670 & -0.335 & -0.335 & 0.670 & -0.335 \\ 0.580 & 0 & -0.580 & 0.580 & 0 & -0.580 \\ 0.474 & 0.474 & 0.474 & 0.474 & 0.474 & 0.474 \\ -15.782 & 15.784 & 31.565 & 15.782 & -15.784 & -31.565 \\ -27.337 & -27.336 & 0.001 & 27.337 & 27.336 & -0.001 \\ 22.319 & -22.319 & 22.319 & -22.319 & 22.319 & -22.319\end{array}\right]$

According to the acceleration matrix of the accelerometer, the theoretical isotropy index can be calculated by Equations (44) - (46) respectively, which is shown in Table 2.

$$
\begin{aligned}
\boldsymbol{G}_{1} & =\left(\boldsymbol{G}_{a 1} \boldsymbol{G}_{\varsigma 1}\right)^{T} \\
S_{a 1} & =\frac{1}{\operatorname{cond}\left(\boldsymbol{G}_{a 1}\right)} \\
S_{\zeta 1} & =\frac{1}{\operatorname{cond}\left(\boldsymbol{G}_{\varsigma 1}\right)}
\end{aligned}
$$

The calibration matrix of the experiment can be calculated by substituting Equation (41) and Equation (42) into Equation (40): 
INTERNATIONAL JOURNAL OF CIRCUITS, SYSTEMS AND SIGNAL PROCESSING

$$
\beta=\left[\begin{array}{cccccc}
0.039 & -0.075 & 0.037 & 0.037 & -0.076 & 0.038 \\
0.065 & -0.001 & -0.067 & 0.066 & 0.001 & -0.065 \\
-0.054 & -0.055 & -0.055 & -0.054 & -0.053 & -0.053 \\
1.769 & -1.769 & -3.549 & -1.781 & 1.781 & 3.564 \\
-3.063 & -3.097 & -0.045 & 3.065 & 3.097 & 0.050 \\
-2.464 & 2.482 & -2.504 & 2.456 & -2.481 & 2.516
\end{array}\right] \times 10^{7}
$$

The calibration matrix reflects the relationship between acceleration and strain, which has the same effect as the acceleration matrix. Hence, the experimental isotropy index can be yielded by the same definition, which is expressed as follows. The results are shown in Table 2.

$$
\begin{aligned}
& \boldsymbol{\beta}=\left(\begin{array}{ll}
\boldsymbol{\beta}_{\alpha} & \boldsymbol{\beta}_{\varsigma}
\end{array}\right)^{T} \\
& S_{a 2}=\frac{1}{\operatorname{cond}\left(\boldsymbol{\beta}_{a 1}\right)} \\
& S_{\varsigma 2}=\frac{1}{\operatorname{cond}\left(\boldsymbol{\beta}_{\varsigma 1}\right)}
\end{aligned}
$$

Table 2. Theoretical and experimental results.

\begin{tabular}{ccc}
\hline No. isotropy index & $S_{a \mathrm{i}}$ & $S_{\varepsilon \mathrm{i}}$ \\
\hline 1 & 0.999 & 1.000 \\
2 & 0.992 & 0.979 \\
\hline
\end{tabular}

where $S_{a i}$ are the linear isotropy index, and $S_{c i}$ are the angular isotropy index. The no. 1 index denotes the theoretical results; the no. 2 index denotes the results of the experiment.

\section{CONCLUSIONS}

The method of circular hyperboloids was used to design the isotropic accelerometer based on modified GSP. The results of isotropy verification and the comparison between the theoretical and experimental isotropy index proved that the method of circular hyperboloids could achieve isotropy. The main conclusions are as follows:

1) In this paper, a novel research method, the method of circular hyperboloids, was applied to study the accelerometer. The static model of the accelerometer was established and the isotropy was defined.

2) By calculating the isotropy index, the method of circular hyperboloids constructed isotropic accelerometer based on equal-leg modified GSP was proven to be reliable.

3) An example was illustrated as to how an isotropic accelerometer was constructed using the method of circular hyperboloids.

4) According to the theory of parallel mechanism and the stiffness on the leg, the relationship between acceleration and strain on each leg was derived. The solid model was constructed by the example's structure. Using the finite element method, the static characteristic was simulated. The theoretical isotropy index and the experimental isotropy index were yielded, respectively. According to the comparison between the theoretical and experimental isotropy index, the method to fabricate the solid model was proven to be reliable.

\section{REFERENCES}

[1] Zou, T.; Angeles, J., Isotropic Accelerometer Strapdowns and Related Algorithms for Rigid-Body Pose and Twist Estimation. Journal of Applied Mechanics 2014, 81, (11), 111003.

[2] Staufer, P.; Gattringer, H., State estimation on flexible robots using accelerometers and angular rate sensors. Mechatronics 2012, 22, (8), 1043-1049.

[3] Wu, L. C.; Nangia, V.; Bui, K., et al., In Vivo Evaluation of Wearable Head Impact Sensors. Annals of Biomedical Engineering 2016, 44, (4), 1234-1245.

[4] Lee, H.; Lee, H.; Whang, M., An Enhanced Method to Estimate Heart Rate from Seismocardiography via Ensemble Averaging of Body Movements at Six Degrees of Freedom. Sensors 2018, 18, (1), 238.

[5] Cui, F.; Liu, W.; Chen, W., et al., Design, Fabrication and Levitation Experiments of a Micromachined Electrostatically Suspended Six-Axis Accelerometer. Sensors 2011, 11, (12), 11206.

[6] Stewart, D., A Platform with Six Degrees of Freedom. ARCHIVE Proceedings of the Institution of Mechanical Engineers 1847-1982 (vols 1-196) 2013, 180, (1965), 371-386.

[7] Gao, Z.; Zhang, D., Design, analysis and fabrication of a multidimension al acceleration sensor based on fully decoupled compliant parallel mecha nism. Sensors \& Actuators A Physical 2010, 163, (1), 418-427.

[8] Sun, Z.; Liu, J.; Yu, C., et al., A Small Range Six-Axis Accelerometer Designed with High Sensitivity DCB Elastic Element. Sensors 2016, 16, (9), 1552.

[9] Sun, Z.; Liu, J.; Yu, C. Z., Research on measuring characteristic for a six-axis accelerometer. Chinese Journal of Scientific Instrument 2016, 37, (2), 467-473.

[10] Yu, C.; Liu, J.; Sun, Z., et al., Research on the dynamic characteristics of parallel six-axis accelerometer based on Kane's method. Chinese Journal of Scientific Instrument 2015, 36, (7), 1611-1617.

[11] Yao, J.; Hou, Y.; Wang, H., et al., Spatially isotropic configuration of Stewart platform-based force sensor. Mechanism \& Machine Theory 2011, 46, (2), 142-155.

[12] Kang, C.-G., Analysis on force sensing errors of force-torque sensors. Trans. Korean Society of Mechanical Engineers 1998, 22, 1250-1257.

[13] Baron, L.; Wang, X.; Cloutier, G. In THE ISOTROPIC CONDITION OF PARALLEL MANIPULATORS OF DELTA TOPOLOGY, Advances in Robot Kinematics, 2002; 2002.

[14] Fattah, A.; Ghasemi, A. M. H., Isotropic Design of Spatial Parallel Manipulators. International Journal of Robotics Research 2002, 21, (9), 811-826.

[15] Tsai, K. Y.; Huang, K. D., The design of isotropic 6-DOF parallel manipulators using isotropy generators. Mechanism \& Machine Theory 2003, 38, (11), 1199-1214.

[16] Zanganeh, K. E.; Angeles, J., Kinematic isotropy and the optimum design of parallel manipulators. The International Journal of Robotics Research 1997, 16, (2), 185-197.

[17] Legnani, G.; Tosi, D.; Fassi, I., et al., The "point of isotropy" and other properties of serial and parallel manipulators. Mechanism \& Machine Theory 2010, 45, (10), 1407-1423.

[18] Wang, H.; Gao, F.; Huang, Z., DESIGN OF 6-AXIS FORCE/TORQUE SENSOR BASED ON STEWART PLATFORM RELATED TO ISOTROPY. Chinese Journal of Mechanical Engineering 1998, 36, (3), 54-59.

[19] Yu, C. Z.; Liu, J.; Sun, Z., Parameters optimization of a six-axis accelerometer based on parallel mechanism. Chinese Journal of Scientific Instrument 2014, 35, (10), 2216-2222.

[20] Afzali-Far, B.; Andersson, A.; Nilsson, K., et al., Dynamic isotropy in 6-DOF kinematically constrained platforms by three elastic nodal joints. Precision Engineering-Journal of the International Societies for Precision Engineering and Nanotechnology 2016, 45, 342-358.

[21] Hou, Y.; Zeng, D.; Yao, J., et al., Optimal design of a hyperstatic Stewart platform-based force/torque sensor with genetic algorithms. Mechatronics 2009, 19, (2), 199-204. 
[22] Jiang, H. Z.; Tong, Z. Z.; He, J. F., Dynamic isotropic design of a class of Gough-Stewart parallel manipulators lying on a circular hyperboloid of one sheet. Mechanism \& Machine Theory 2011, 46, (3), 358-374.

[23] Tong, Z.; Jiang, H.; Jingfeng, H. E., Optimal Design of Isotropy Performance of Six-dimensional Force Sensor Based on Standard Stewart Parallel Structure Lying on a Circular Hyperboloid of One Sheet. Acta Aeronautica Et Astronautica Sinica 2011, 32, (12), 2327-2334.

[24] Bandyopadhyay, S.; Ghosal, A., An algebraic formulation of kinematic isotropy and design of isotropic 6-6 Stewart platform manipulators. Mechanism \& Machine Theory 2008, 43, (5), 591-616.

[25] Yao, J., Analytic Analysis and Optimal Design of Isotropy Performance of Six-dimensional Force Sensor Based on Stewart Platform. Journal of Mechanical Engineering 2009, 45, (12), 22-28.

[26] Tong, Z.; Jiang, H.; Jingfeng, H. E., et al., Optimal Design of Acceleration Sensor Based on Generalized Gough-Stewart Parallel Manipulator Lying on Pair of Circular Hyperboloids. Journal of Mechanical Engineering 2014, 50, (13), 35.[Online]. Available: http://www.cjmenet.com.cn/Jwk \jxgcxb/CN/Y2014/V50/I13/35.

[27] Jafari, F.; Mcinroy, J. E., Orthogonal Gough-Stewart platforms for micromanipulation. Robotics \& Automation IEEE Transactions on 2003, 19, (4), 595-603.

[28] Hou, Y.; Yao, J.; Lu, L., et al., Performance analysis and comprehensive index optimization of a new configuration of Stewart six-component force sensor. Mechanism \& Machine Theory 2009, 44, (2), 359-368.

[29] Jiang, H. Z.; He, J. F.; Tong, Z. Z., et al., Dynamic isotropic design for modified Gough-Stewart platforms lying on a pair of circular hyperboloids. Mechanism \& Machine Theory 2011, 46, (9), 1301-1315.

[30] Knight, B. F. Deployable antenna kinematics using tensegrity structure design. Ph.D. Thesis, University of Florida, Florida, the United States, 2000.

[31] Merlet, J. P., Jacobian, Manipulability, Condition Number and Accuracy of Parallel Robots. Journal of Mechanical Design 2006, 128, (128), 199-206.

[32] Yu, C. Z. Study on a six-axis accelerometer based on stewart platform. Ph.D. Thesis, Beijing University of Posts and Telecommunications, Beijing, 2012.

[33] Uchiyama, M.; Nakamura, Y.; Hakomori, K., Evaluation of the robot force sensor structure using singular value decomposition. Advanced Robotics 2012, volume 5, (5), 39-52.

[34] Yao, J.; Hou, Y.; Wang, H., et al. In Isotropic design of Stewart platform-based force sensor, International Conference on Intelligent Robotics and Applications, 2008; Springer: 2008; pp 723-732.

[35] Yao, J. Basic theory and expermental research on large range isotropy performance of a six-dimensional force sensor. Ph.D. Thesis, Yanshan University, Qin Huangdao, 2010.

[36] Zhao, Y.-S.; Hou, Y.-L.; Yan, Z.-W., et al. In Research and design of a pre-stressed six-component force/torque sensor based on the Stewart platform, ASME 2005 International Design Engineering Technical Conferences and Computers and Information in Engineering Conference, 2005; American Society of Mechanical Engineers: 2005; pp 573-581.

[37] Li, H.; Hao, G., Constraint-force-based approach of modelling compliant mechanisms: Principle and application. Precision Engineering 2017, 47, 158-181.

[38] Hao, G.; Murphy, M.; Luo, X. In Development of a compliant-mechanism-based compact three-axis force sensor for high-precision manufacturing, ASME 2015 International Design Engineering Technical Conferences and Computers and Information in Engineering Conference, 2015; American Society of Mechanical Engineers: 2015; pp V009T07A082-V009T07A082.

[39] Hao, G.; Kong, X., A structure design method for compliant parallel manipulators with actuation isolation. Mechanical Sciences 2016, 7, (2), 247. [Online]. Available: https://doi.org/10.5194/ms-7-247-2016
Creative Commons Attribution License 4.0 (Attribution 4.0

International, CC BY 4.0)

This article is published under the terms of the Creative Commons

Attribution License 4.0

https://creativecommons.org/licenses/by/4.0/deed.en_US 